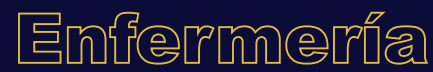

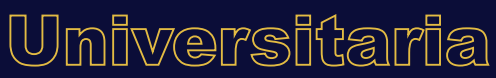

\section{Incertidumbre y afrontamiento en familiares de personas dependientes del alcohol: apoyo social y espiritualidad}

\author{
Uncertainty and coping among \\ relatives of alcohol dependent persons: \\ social support and spirituality
}

\section{Incerteza e enfrentamento em parentes de pessoas dependentes de álcool: apoio social e espiritualidade}

L. Hinojosa-García ${ }^{a 1}$, M.M. Alonso-Castillo ${ }^{b_{2} *}$, P. García-García ${ }^{\mathrm{c}}$, A.A. Jiménez-Martínez ${ }^{\mathrm{d} 1}$, N.A. Armendáriz-García ${ }^{\mathrm{e} 2}$

ORCID

${ }^{\mathrm{a}}$ 0000-0002-4473-9275

${ }^{\mathrm{d}} 0000-0001-8679-0194$

${ }^{\mathrm{b}} 00000-0002-7197-8116$

e $0000-0001-9033-3244$

c0000-0003-0929-2807

${ }^{1}$ Universidad Autónoma de Tamaulipas, Unidad Académica Multidisciplinaria Matamoros, H. Matamoros, Tamaulipas, México

${ }^{2}$ Universidad Autónoma de Nuevo León, Facultad de Enfermería, Monterrey, Nuevo León, México

Recibido: 27 enero 2020

Aceptado: 19 agosto 2020

RESUMEN

Introducción: La dependencia al alcohol, considerada como una enfermedad crónica, es un detonante de incertidumbre para el familiar de la persona con dependencia, lo cual genera trastornos emocionales negativos. El apoyo social y la espiritualidad son mecanismos de

*Autora para correspondencia. Correo electrónico: magdalena_alonso@hotmail.com https://doi.org/10.22201/eneo.23958421e.2020.4.828

1665-7063/@ 2020 Universidad Nacional Autónoma de México, Escuela Nacional de Enfermería y Obstetricia. Este es un artículo Open Access bajo la licencia CC BY-NC-ND (http://creativecommons.org/licenses/by-nc-nd/4.o/). 
afrontamiento que podrían disminuir la incertidumbre y favorecer el bienestar tanto físico, como psicológico.

Objetivos: Determinar la relación y el efecto del apoyo social y la espiritualidad sobre la incertidumbre del familiar de la persona dependiente del alcohol.

Métodos: Estudio descriptivo y correlacional, muestra de 135 mujeres pertenecientes a grupos Al-Anon, se utilizaron la Escala de Percepción de Incertidumbre en Padres y Miembros de la Familia (PPUS-FM) de Mishel, el cuestionario MOS-SSS de Sherbourne y Stewart y la Escala de Perspectiva Espiritual elaborada por Reed.

Resultados: Se identificó un coeficiente de correlación negativo y significativo de la incertidumbre con el apoyo social $\left(r_{s}=-.356, p<.01\right)$ y la espiritualidad $\left(r_{s}=-.216, p<.05\right)$. Mediante un modelo de regresión lineal general univariado se determinó que únicamente el apoyo social influye en la disminución de la incertidumbre $(\beta=-.280, t=-3.62, p=.001)$ con un coeficiente de determinación o varianza explicada del 11.5\%, mientras que la espiritualidad no mostró efecto $(\beta=-.041, t=.218, p>.05)$ sobre la incertidumbre.

Conclusiones: El apoyo social es identificado como un mecanismo de afrontamiento efectivo que permitirá al personal de enfermería desarrollar intervenciones dirigidas a reducir la incertidumbre en familiares de personas con algún tipo de dependencia.

Palabras clave: Incertidumbre; afrontamiento; apoyo social; espiritualidad; México.

\section{ABSTRACT}

Introduction: Alcohol dependency is a chronic illness which generates uncertainty and other emotional problems among the relatives of persons who suffer alcoholism. Social support and spirituality are some coping mechanisms which could reduce uncertainty and favor the physical and psychological wellbeing.

Objective: To determine the relationship and the effect of social support and spirituality on the uncertainty experienced by relatives of alcohol dependent persons.

Methods: This is a descriptive and correlational study. The sample was constituted by 135 women members of Al-Anon. The Mishel Scale of Perception of Uncertainty among Parents and Family Members (PPUS-FM), the Sherbourne and Stewart Questionnaire, and the Reed Spiritual Perspective Scale were all used.

Results: Significant negative correlation coefficients between uncertainty and social support $\left(r_{s}=-.356, p .05\right)$. and spirituality $\left(r_{s}=-.216, p\right.$.05). were identified. Using a univariate general linear regression model, it was determined that only social supports reduced uncertainty $(\beta=-.280, t=-3.62, p=.001)$ with $11.5 \%$ of variance explained; while spirituality did not produce any effect $(\beta=-.041, t=.218, p>.05)$.

Conclusions: Social support is identified as a coping mechanism which can be used by nursing staff to develop interventions aimed at reducing uncertainty among relatives of alcohol dependent persons.

Keywords: Uncertainty; coping; social support; spirituality; Mexico.

RESUMO

Introdução: A dependência do álcool, considerada como uma doença crónica, é um gatilho de incerteza para o familiar da pessoa com dependência, o qual gera transtornos emocionais 
negativos. O suporte social e a espiritualidade são mecanismos de enfrentamento que poderiam diminuir a incerteza e favorecer o bem-estar tanto físico, quanto psicológico.

Objetivos: Determinar a relação e o efeito do suporte social e a espiritualidade sobre a incerteza do familiar da pessoa dependente de álcool.

Métodos: Estudo descritivo e correlacional, amostra de 135 mulheres pertencentes aos grupos Al-Anon, utilizaram-se a Escala de Percepção de Incerteza em Pais e Membros da Família (PPUS-FM) de Mishel, o questionário MOS-SSS de Sherbourne e Stewart e a Escala de Perspectiva Espiritual elaborada por Reed.

Resultados: Identificou-se um coeficiente de correlação negativo e significativo da incerteza com o apoio social $\left(r_{s}=-.356, p<.01\right)$ e a espiritualidade $\left(r_{s}=-.216, p<.05\right)$. Mediante um modelo de regressão linear geral univariado determinou-se que unicamente o apoio social influencia a diminuição da incerteza $(\beta=-.280, t=-3.62, p=.001)$ com um coeficiente de determinação ou variância explicada do $11.5 \%$, enquanto a espiritualidade não mostrou efeito ( $\beta=-.041$, $t=.218, p>$.05) sobre a incerteza.

Conclusões: O suporte social é identificado como um mecanismo de enfrentamento eficaz que permitirá ao pessoal de enfermagem desenvolver intervenções dirigidas a reduzir a incerteza em familiares de pessoas com algum tipo de dependência.

Palavras chave: Incerteza; enfrentamento; apoio social; espiritualidade; México.

\section{INTRODUCCIÓN}

El concepto de incertidumbre ha sido definido de distintas formas; comúnmente refiere a la situación de desconocimiento que se tiene acerca de lo que sucederá en el futuro, a la falta de seguridad, confianza o de certeza sobre algo que crea inquietud. Para la Real Academia Española ${ }^{1}$ el término incertidumbre hace mención a la falta de certidumbre.

En la Teoría de Rango Medio (TRM) Incertidumbre ante la enfermedad, Mishel la define como un estado cognitivo caracterizado por la inhabilidad de la persona para estructurar o determinar el significado de los hechos o acontecimientos relacionados a una enfermedad, lo que permite que el individuo no pueda dar valores definidos a los hechos, y por ende no pueda predecir con precisión los resultados, todo esto motivado por la falta de información y conocimiento². El marco estructural de la teoría se compone de tres elementos principales: antecedentes, valoración y afrontamiento de la incertidumbre.

La incertidumbre puede ser valorada a través de la inferencia de un peligro e implicar un daño emocional y físico. También ha sido identificada como un tipo de respuesta que puede estar presente tanto en los pacientes, como en los familiares o cuidadores de personas con enfermedades crónicas ${ }^{3.4}$. Además, genera trastornos emocionales negativos como estrés, ansiedad, angustia, irritabilidad, por lo que de no contar con mecanismos de afrontamiento podría afectar el bienestar psicológico y físico de las personas y repercutir en una disminución de su calidad de vida 5 .

Por otro parte, la dependencia al alcohol se considera una enfermedad crónica, recurrente, que se deriva de un consumo sostenido de alcohol, la cual tiene consecuencias agudas y crónicas y se asocia con más de doscientas enfermedades y trastornos ${ }^{6}$. La presencia de la dependencia al alcohol en un miembro de la familia es el detonante de la incertidumbre del familiar principal, figura representada generalmente por la esposa, madre, hermanas e hijas de la persona consumidora. Esta condición se deriva de la falta de información, imprevisibilidad, inseguridad y la duda acerca de los eventos y 
circunstancias que rodean la enfermedad de su familiar, así como del futuro del consumidor y de la familia en general.

En una de las proposiciones de su TRM Mishel señala: "la incertidumbre valorada como un peligro, provoca una serie de mecanismos de afrontamiento dirigidos a reducir la incertidumbre" ${ }^{2}$. En este sentido, la literatura documenta que cuando la incertidumbre es valorada como peligro, la espiritualidad y el apoyo social actúan como mecanismos de afrontamiento efectivos para su reducción y coadyuvan en la disminución de las alteraciones emocionales del cuidador o familiar principal de las personas con enfermedades crónicas ${ }^{7-9} \mathrm{y}$, por lo tanto, se mejora su bienestar físico y emocional.

De acuerdo a Reed, la espiritualidad es la experiencia interna del ser humano que se evidencia a través de la autoconciencia, las prácticas, las creencias y las necesidades espirituales. Asimismo, la espiritualidad se refiere a la predisposición para construir significados a través de un sentido de conexión con un ser de naturaleza superior o a la existencia de un propósito supremo, superior a sí mismo ${ }^{10}$. Por ejemplo, dentro de los grupos de ayuda mutua Al-Anon -no aliados a ningún tipo de religión- se utilizan recursos espirituales diversos como la oración, la lectura espiritual y la fe en un ser supremo. El apoyo social se expresa mediante la ayuda, apoyo, consejo, afecto, fortaleza y esperanza que recibe el familiar del dependiente del alcohol al participar en estos grupos.

La teoría de Mishel ha sido utilizada en el estudio de la incertidumbre en pacientes con desórdenes temporomandibulares ${ }^{11}$, con traumatismo de la médula espinal ${ }^{12}$, en pacientes con lupus eritematoso ${ }^{13}$, fibromialgia ${ }^{14}$, cáncer de mama ${ }^{15}$ y diabetes mellitus tipo $2^{16}$, en cuidadores familiares de pacientes hospitalizados en unidades de cuidado intensivo ${ }^{17}$ y acompañantes de pacientes en servicios de urgencias 3 . Sin embargo, no se identificó hasta el momento actual literatura que valore los mecanismos de afrontamiento empleados ante la incertidumbre en pacientes con algún tipo de adicción, así como en cuidadores o familiares principales de éstos, lo que revela un vacío de conocimiento. Por consiguiente, se precisa indagar en relación a esta temática dada la magnitud creciente de la dependencia al alcohol, además del sufrimiento e impacto doloroso que tiene en la familia, por lo cual se plantearon los siguientes objetivos de estudio:

1. Determinar la relación de la incertidumbre con el apoyo social y la espiritualidad del familiar principal de la persona dependiente del alcohol.

2. Identificar el efecto del apoyo social y la espiritualidad sobre la incertidumbre del familiar principal de la persona dependiente del alcohol.

\section{METODOLOGÍA}

Se trata de un estudio de diseño cuantitativo, descriptivo, correlacional y transversal, la población estuvo conformada por familiares de personas dependientes del alcohol que participaban en grupos Al-Anon de la frontera norte de México, el muestreo fue de tipo censal (grupos Al-Anon) para una muestra final de 135 participantes en el estudio, todas ellas pertenecientes al sexo femenino. Para la obtención de los datos fueron utilizadas una cédula de datos personales, además de tres instrumentos.

Para medir la incertidumbre se utilizó la Escala de Percepción de Incertidumbre en Padres y Miembros de la Familia (PPUS-FM por sus siglas en inglés) de Mishel ${ }^{18}$. La escala está conformada por 31 reactivos en una escala tipo likert con cinco opciones de respuesta, 1 equivale a totalmente en desacuerdo, 2 desacuerdo, 3 indeciso, 4 de acuerdo y 5 totalmente de acuerdo. La escala tiene como puntaje global máximo 155 puntos y mínimo 31; donde a mayor puntaje mayor nivel de incertidumbre. 
Para este análisis fueron utilizados 28 reactivos en virtud de la temática del estudio, por lo que la escala se evaluó de la siguiente forma: el puntaje global máximo fue de 140 puntos y el mínimo de 28; donde a mayor puntaje, mayor incertidumbre. Los niveles de incertidumbre consideraron tres puntos de corte y fueron clasificados de la siguiente forma: bajo nivel de incertidumbre < 53 puntos, regular nivel de incertidumbre entre 53 y 84 puntos y alto nivel de incertidumbre $>84$ puntos. El instrumento ha sido utilizado en población mexicana en padres de niños con cáncer, dónde se reportó un alfa de Cronbach de $0.90^{19}$. Para el presente estudio el Coeficiente de Confiabilidad fue de o.9o.

El apoyo social fue medido a través del cuestionario MOS-SSS (Medical Outcomes StudySocial Support Survey) de Sherbourne y Stewart ${ }^{20}$. La escala mide el apoyo social en su dimensión estructural (el tamaño de la red social) y funcional (la utilidad que ésta tiene). El instrumento consta de veinte reactivos donde el primer reactivo se evalúa con porcentajes, el resto del instrumento se presenta en una escala likert con cinco opciones de respuesta que van desde nunca (con un valor de 1) hasta siempre (con valor de 5), el puntaje global de apoyo máximo es de 95, con valor medio de 57 y un mínimo de 19, entre mayor sea la puntuación, mayor es el apoyo social percibido. En México fue utilizado para medir el apoyo social en adultos mayores, mostrando un alfa de Cronbach de $0.96^{21}$. En este estudio el Coeficiente de Confiabilidad fue de 0.95 .

La variable espiritualidad fue medida por medio de la Escala de Perspectiva Espiritual, elaborada por Reed $^{10}$, para medir la visión espiritual de una persona y las interacciones relacionadas con la espiritualidad. Consta de diez enunciados que se califican en un rango de 1 a 6 para un puntaje total de 60 , donde a mayor puntaje mayor espiritualidad. Las cuatro primeras preguntas se responden con los siguientes criterios: 1) Nunca, 2) Menos de una vez al año, 3) Más o menos una vez al año, 4) Más o menos una vez al mes, 5) Más o menos una vez a la semana y 6) Más o menos una vez al día. Las preguntas del 5 al 10 son respondidas de la siguiente manera: 1) Extremadamente en desacuerdo, 2) Desacuerdo, 3) En desacuerdo más que en acuerdo, 4) De acuerdo más que en desacuerdo, 5) De acuerdo y 6) Extremadamente de acuerdo. El instrumento consta de una subescala denominada prácticas espirituales (1 al 4), y otra subescala denominada creencias espirituales (del 5 al 10); ambas se califican en un rango de 1 a 6 , con un puntaje total de 24 para la primera subescala y 36 la segunda. El puntaje de la escala total oscila entre 10 a 60 puntos, donde a mayor puntaje mayor espiritualidad. El instrumento ha reportado alfa de Cronbach en población mexicana de $0.88^{22}$. Para el presente estudio el Coeficiente de Confiabilidad fue de 0.87.

El estudio fue aprobado por la Comisión de Investigación y Ética de la Facultad de Enfermería de la Universidad Autónoma de Nuevo León, México (FAEN-D-1204), además se contó con la autorización de la coordinadora de cada uno de los grupos Al-Anon participantes ubicados en la frontera noroeste de México. Los datos fueron colectados en el lugar dónde habitualmente tienen lugar sus sesiones. El estudio se apegó a lo dispuesto por el Reglamento de la Ley General de Salud en materia de Investigación para la Salud ${ }^{23}$, en el cual se establece que para el desarrollo de investigación en salud se deben contemplar aspectos éticos que garanticen la dignidad, consentimiento, confidencialidad, anonimato y el bienestar de los individuos.

Para el análisis de los datos se utilizó el paquete estadístico Statistical Package for the Social Sciences (SPSS) versión 21. Se revisó la consistencia interna de los instrumentos a través del Coeficiente de Confiabilidad de Alfa de Cronbach, posteriormente se aplicó la Prueba de Bondad de Ajuste de Kolmogorov-Smirnov con corrección de Lilliefors para decidir el uso de pruebas estadísticas paramétricas o no paramétricas. En razón de que las variables de estudio no presentaron 
normalidad ( $p<.05)$, se decidió el uso de pruebas no paramétricas por medio de la prueba correlación de Spearman, además de un modelo de regresión lineal general univariado para identificar si existía relación y efecto entre las variables de estudio.

\section{RESULTADOS}

Los datos sociodemográficos del total de la muestra conformada por 135 mujeres revelan que el mayor porcentaje de ellas (31.1\%) se ubica en el rango de los 51 a 60 años de edad. En relación al nivel de escolaridad, $24 \%$ de las participantes cuenta con estudios de preparatoria, seguido por el nivelsecundaria(23\%),mientrasqueunsignificativo porcentaje(20.0\%)cuentaconestudiosprofesionales. En cuanto a la ocupación, 56.3\% trabajan, 25.9\% son amas de casa y solo 6.7\% mencionaron estudiar y trabajar, mientras que el mayor porcentaje de la muestra mencionó profesar la religión católica (70.4\%). Se encontró que el total de las participantes presentaban algún nivel de incertidumbre, predominando el nivel alto 58.5\% (79), nivel regular 35.6\% (48) y nivel bajo 5.9\% (8). El número de amigos cercanos (muchos de los cuales se encuentran dentro de los grupos de ayuda mutua AlAnon) se relacionó negativa y significativamente con el índice de incertidumbre $\left(r_{\mathrm{s}}=-.270, p<.01\right)$.

Para responder al primer objetivo del estudio, es decir, determinar la relación de la incertidumbre con el apoyo social y la espiritualidad del familiar principal de la persona dependiente del alcohol, fue realizada la prueba de correlación bivariada de Spearman. En la tabla 1 es posible observar que la variable incertidumbre se correlaciona de forma negativa y significativa tanto con el apoyo social $\left(r_{\mathrm{s}}=-.356, p<.01\right)$; como con la espiritualidad $\left(r_{\mathrm{s}}=-.216, p<.05\right)$, en otras palabras, cuanto mayor sea el apoyo social y la espiritualidad, menor es la incertidumbre del familiar de la persona con dependencia al alcohol.

De igual forma, se aplicó un modelo de regresión lineal general univariado para identificar el efecto del apoyo social y la espiritualidad sobre la incertidumbre como variable dependiente. Los resultados muestran que el modelo fue significativo, explicando que la incertidumbre es influida únicamente por el apoyo social ( $\beta=-.280, t=-3.62, p=.001)$ con un coeficiente de determinación o varianza explicada del 11.5\%, mientras que la espiritualidad no mostró efecto ( $\beta=-.041, t=.218, p>.05)$ sobre la incertidumbre. Además, el coeficiente Bootstrap reporta significancia para el apoyo social, por lo que se reafirma que la variable apoyo social tiene un efecto sobre la incertidumbre (Tabla 2).

\section{DISCUSIÓN}

El presente estudio permitió evidenciar relación y efecto significativo de la variable apoyo social sobre la incertidumbre, la cual es consistente con lo señalado por Mishel $^{2}$ en su teoría de la incertidumbre ante la enfermedad, al indicar que el apoyo social se relaciona negativamente con ésta y actúa como un favorecedor en la reducción de la incertidumbre tanto de los pacientes, como en los familiares de las personas con enfermedades crónicas.

De igual forma, los hallazgos son similares a la literatura revisada, donde identifican al apoyo social y las redes de apoyo social conformadas por familiares, amigos, vecinos o grupos de ayuda 
Tabla 2. Efecto del apoyo social y la espiritualidad sobre la incertidumbre

\begin{tabular}{lrrrrr} 
Modelo & SC & gl & CM & $\boldsymbol{F}$ & $P$ \\
\hline Apoyo social & 3846.947 & 1 & 3846.947 & 13.134 & .001 \\
\hline Espiritualidad & 13.908 & 1 & 13.908 & .047 & .828 \\
\hline
\end{tabular}

$R^{2}$ apoyo social $=11.5 ; R^{2}$ espiritualidad $=2.7$

\begin{tabular}{lccrc} 
Modelo & \multicolumn{5}{c}{ Coeficientes } \\
\cline { 2 - 5 }$(\mathrm{n}=135)$ & $\boldsymbol{B}$ & $\boldsymbol{E}$ & $\boldsymbol{t}$ & $\boldsymbol{P}$ \\
\hline Constante & & & -3.624 & .001 \\
\hline Apoyo social & -.280 & .077 & .218 & .828 \\
\hline Espiritualidad & -.041 & .188 & &
\end{tabular}

\section{Bootstrap para coeficientes}

\begin{tabular}{llcccc}
\hline & & & \multicolumn{2}{c}{ IC 95\% } \\
\hline Variable dependiente & Parámetro & $\boldsymbol{B}$ & $\boldsymbol{p}$ & LI & LS \\
\hline Incertidumbre & Apoyo social & -.282 & .001 & -.435 & -.128 \\
\hline Incertidumbre & Espiritualidad & -.043 & .818 & -.414 & -.328 \\
\hline
\end{tabular}

Nota: $R^{2}=$ coeficiente de determinación, $S C$ = suma de cuadrados, $g l=$ grados de libertad, $C M=$ cuadrado medio, $\beta=$ Beta, $\mathrm{p}$ = significancia, $E=$ Error típico, $t=$ estadístico de la prueba $t$

mutua, como un importante recurso para afrontar positivamente las crisis e incertidumbre generadas por desconocimiento acerca de la enfermedad, la ambigüedad, la falta de claridad e información y la imprevisibilidad, así como los eventos acerca de la enfermedad evaluados como peligrosos ${ }^{6,24,25}$. Para los familiares principales de estos pacientes dependientes del alcohol, que en su mayoría suelen ser mujeres, el contar con el apoyo de familiares, amigos, vecinos o compañeras de los grupos de ayuda mutua como Al-Anon, representa una ventana de oportunidad para compartir sus experiencias, tomar distancia de la problemática y recibir consejo, orientación y ayuda para afrontar la incertidumbre de vivir con una persona con dependencia al alcohol ${ }^{26}$.

Otro recurso de afrontamiento identificado en el presente estudio fue la espiritualidad, hecho que es congruente con lo reportado en estudios previos, donde señalan que cuando las personas se enfrentan a enfermedades crónicas incrementan sus prácticas y creencias espirituales ${ }^{22}$, buscando la intervención y protección de un ser divino que cambie su situación ${ }^{27}$. Por consiguiente, se vuelve necesario que los profesionales de enfermería identifiquen la influencia que tiene la espiritualidad sobre la calidad de vida, además de reconocer el valor que tienen los grupos de apoyo espiritual para prevenir la aparición o modular la incertidumbre del familiar principal o cuidador ante la enfermedad ${ }^{28}$.

\section{CONCLUSIONES}

Con base en los resultados obtenidos en el presente estudio, y de acuerdo a la literatura revisada, se puede concluir que, tanto los pacientes, como los familiares principales o cuidadores de personas que tienen una enfermedad crónica (dentro de las cuáles es considerada la dependencia al alcohol), muestran un alto riesgo de experimentar incertidumbre, derivada del desconocimiento acerca de la 
enfermedad, las experiencias negativas vividas, los cambios sufridos en la dinámica familiar y el no saber o no contar con los recursos necesarios para afrontarlo.

Ante esto, es imprescindible que los profesionales de enfermería identifiquen las redes de apoyo social disponibles en la comunidad, dentro de las cuales se encuentran los grupos de ayuda mutua Al-Anon, donde gracias a los aportes que reciben de su inclusión, resultan ser un mecanismo efectivo de afrontamiento para los familiares principales de personas que abusan de algún tipo de sustancia. El ampliar el conocimiento del curso de la enfermedad permite a los familiares o cuidadores de la persona dependiente del alcohol realizar cambios en su vida, les ayuda a valorarse como persona, coadyuva a eliminar estereotipos, a saber perdonar y perdonarse, a poner límites, además de concientizarse de que hay otras formas más saludables de vivir y relacionarse, facilitando con todo esto, el disminuir su incertidumbre y en consecuencia, mejorar su bienestar psicológico y su calidad de vida. Por último, se recomienda continuar investigando sobre la problemática de la dependencia al alcohol y su impacto en las personas, familias y comunidades.

\section{RESPONSABILIDADES ÉTICAS}

Protección a personas y animales. En este estudio no se realizaron experimentos con personas o animales.

Confidencialidad de los datos. Se garantizó la confidencialidad y anonimato de las personas participantes.

Conflicto de intereses. Los autores declaran no tener conflicto de intereses.

Financiamiento. Ninguno.

\section{REFERENCIAS}

1. Real Academia Española. Diccionario de la lengua española. Incertidumbre. Madrid: RAE; 2021.

2. Mishel MH. Reconceptualization of the uncertainty in illness theory. Image J Nurs Sch. 1990; 22(4): 256-62. https://doi.org/10.1111/j.1547-5069.1990.tboo225.x

3. Brito-Brito PR, García-Tesouro E, Fernández-Gutiérrez DA, García-Hernández AM, Fernández-Gutiérrez R, Burillo-Putze G. Validación de la Escala de Incertidumbre ante la Enfermedad en pacientes y acompañantes que acuden a un servicio de urgencias. Emergencias. 2018; 30: 105-14. https://bit.ly/2PoxM5P

4. Flórez-Torres IER, Montalvo-Prieto A, Romero-Massa E. Asociación entre el nivel de incertidumbre y el tipo de enfermedad en pacientes hospitalizados en UCI. av. enferm. 2018; 36(3): 283-91.

https://doi.org/10.15446/av.enferm.v36n3.66508

5. Etkind SN, Bristowe K, Bailey K, Selman LE, Murtagh FEM. How does uncertainty shape patient experience in advanced illness? A secondary analysis of qualitative data. Palliative Medicine. 2017; 31(2): 171-80. https://doi.org/10.1177/0269216316647610

6. Organización Mundial de la Salud. Alcohol. Datos y cifras. Ginebra: OMS; 2018. https://bit.ly/39d2V2F

7. Arteaga-Noriega A, Cogollo-Jiménez R, Muñoz-Monterroza D. Apoyo social y control metabólico en la diabetes mellitus tipo 2. Rev Cuid. 2017; 8(2): 1668-76.

https://doi.org/10.15649/cuidarte.v8i2.405

8. Hoth KF, Wamboldt FS, Ford DW, Sandhaus RA, Strange C, Bekelman DB, et al. The social environment and illness uncertainty in chronic obstructive pulmonary disease. Int J Behav Med. 2015; 22(2): 223-32. https://doi.org/10.1007/s12529-014-9423-5 
9. Iranmanesh S, Tirgari B, Tofighi M, Forouzi MA. Spiritual wellbeing and perceived uncertainty in patients with multiple sclerosis in south-east Iran. Int J Palliat Nurs. 2014; 20(10): 483-92.

https://doi.org/10.12968/ijpn.2014.20.10.483

10. Reed PG. Developmental resources and depression in the elderly. Nurs Res. 1986; 35(6): 368-74. https://bit.ly/2NSsOxl

11. Yang D, Ye J, Zhou F, Li J, Huang Q, Wan L. Relationship between uncertainty in illness, mood state and coping style in patients with temporomandibular disorders. Int J Nurs Sci. 2015; 2(4); 361-5. https://doi.org/10.1016/j.ijnss.2015.11.003

12. Burbano-López C, Sánchez LE. Traumatismo de la médula espinal e incertidumbre desde la teoría de Merle Mishel. Enferm. univ. 2017; 14(3): 176-83.

https://doi.org/10.1016/j.reu.2017.06.004

13. Lazo-Sánchez MM, Cadena-Estrada JC, Olvera-Arreola SS. Atención de enfermería a una persona con lupus eritematoso sistémico basado en la teoría de incertidumbre. Rev. mex. enferm. cardiol. 2018; 26(3): 92-101. https://bit.ly/3w15FKi

14. Triviño-Martínez A, Solano-Ruiz MC, Siles-González J. Aplicación del modelo de incertidumbre a la fibromialgia. Aten Primaria. 2016; 48(4): 219-25. https://doi.org/10.1016/j.aprim.2015.04.007

15. Ramírez-Perdomo CA, Rodríguez-Velez ME, Perdomo-Romero AY. Incertidumbre frente al diagnóstico de cáncer. Texto contexto-enferm. 2018; 27(4): 1-9.

https://doi.org/10.1590/0104-07072018005040017

16. Gaxiola-Flores M, Herrera-Paredes JM, Álvarez-Aguirre A, Ruiz-Recendiz MJ. Revisión del efecto de la incertidumbre en personas adultas con diabetes mellitus tipo 2. Rev Enferm Inst Mex Seguro Soc. 2019; 27(2): 105-15. https://bit.ly/2QIjAVC

17. Flórez-Torres IE, Montalvo-Prieto A, Romero-Massa E. Incertidumbre en cuidadores familiares de pacientes hospitalizados en unidades de cuidado intensivo. Investig. enferm. imagen desarro. 2018; 20(1): 1-12. https://doi.org/10.11144/Javeriana.ie2O-1.icfp

18. Mishel MH. Uncertainty in acute illness. Annu Rev Nurs Res. 1997; 15, 57-80. https://bit.ly/3tWLvzj

19. Nájera-Gutiérrez G, Ledezma-Martínez MV, Patiño-Meza JN, Galicia-Aguilar RM. Incertidumbre en padres de niños con cáncer. Enf Neurol. 2010; 9(1): 2O-2. https://bit.ly/3rt70oe

20. Sherbourne DC, Stewart LA. The MOS social support survey. Soc. Sci. Med. 1991; 32(6): 705-14. https://doi.org/10.1016/0277-9536(91)90150-B

21. Ugarte-Esquivel A, Morales-Jinez A, López-Rincón FJ, Noris-Vargas M, Rodríguez-Mejía LE, CerdaFlores RM. Apoyo social y su influencia en los niveles de percepción de la salud y depresión en adultos mayores, no hospitalizados, de Torreón Coahuila México. Cuba:XVI Coloquio Panamericano de Investigación en Enfermería; 2018.

22. Jasso-Soto ME, Pozos-Magaña MG, Cadena-Estrada JC, Olvera-Arreola SS. Calidad de vida y perspectiva espiritual de los pacientes hospitalizados con enfermedad cardiovascular. Rev Enferm Inst Mex Seguro Soc. 2017; 25(1): 9-17. https://bit.ly/3firAsd

23. Secretaría de Salud. Reglamento de la ley general de salud en materia de investigación para la salud. México: SSA; Última reforma 2014.

24. Amaya-Ropero MC, Carrillo-González GM. Apoyo social percibido y afrontamiento en personas con dolor crónico no maligno. Aquichan. 2015; 15(4): 461-74.

https://doi.org/10.5294/aqui.2015.15.4.2 
25. García FE, Manquián E, Rivas G. Bienestar psicológico, estrategias de afrontamiento y apoyo social en cuidadores informales. Psicoperspectivas. 2016; 15(3): 101-11.

https://doi.org/10.5027/psicoperspectivas-Vol15-Issue3-fulltext-770

26. Hinojosa-García L, Alonso-Castillo MM, Villar-Luis MA. Experiencias de mujeres familiares de dependientes del alcohol de grupos de ayuda mutua Al-Anon. Rev enferm Herediana. 2017; 10(1): 42-8. https://doi.org/10.20453/renh.v10i1.3130

27. Bautista-Espinel GO, Velandia-Galvis ML, Arévalo-Rangel JA Amaya-Quintero C, Bayona-Pérez $\mathrm{N}$. Incertidumbre en los cuidadores de niños hospitalizados en unidades de: cuidado intensivo neonatal-cuidado intensivo pediátrico (UCIN-UCIP). Cult. cuid. 2019; 23(55): 256-69.

https://doi.org/10.14198/cuid.2019.55.22

28. Arias-Rojas M, Carreño-Moreno S, Posada-López C. Incertidumbre de cuidadores familiares ante la enfermedad de pacientes en cuidados paliativos y factores asociados. Rev. Latino-Am. Enfermagem. 2019; 27: 1-8. https://doi.org/10.1590/1518-8345.3185.3200 